\title{
Activity of Renshaw Cells during Locomotor-Like Rhythmic Activity in the Isolated Spinal Cord of Neonatal Mice
}

\author{
Hiroshi Nishimaru, ${ }^{1,2}$ Carlos E. Restrepo, ${ }^{1}$ and Ole Kiehn ${ }^{1}$ \\ ${ }^{1}$ Mammalian Locomotor Laboratory, Department of Neuroscience, Karolinska Institute, Stockholm S-171 77, Sweden, and ${ }^{2}$ Neuroscience Research \\ Institute, National Institute of Advanced Industrial Science and Technology, Tsukuba 305-8566, Japan
}

In the present study, we examine the activity patterns of and synaptic inputs to Renshaw cells (RCs) during fictive locomotion in the newborn mouse using visually guided recordings from GABAergic cells expressing glutamic acid decarboxylase 67-green fluorescent protein (GFP). Among the GFP-positive neurons in the lumbar ventral horn, RCs were uniquely identified as receiving ventral rootevoked short-latency EPSPs that were markedly reduced in amplitude by nicotinic receptor blockers mecamylamine or tubocurarine. During locomotor-like rhythmic activity evoked by bath application of 5-HT and NMDA, 50\% of the recorded RCs fired in-phase with the ipsilateral L2 flexor-related rhythm, whereas the rest fired in the extensor phase. Each population of RCs fired throughout the corresponding locomotor phase. All RCs received both excitatory and inhibitory synaptic inputs during the locomotor-like rhythmic activity. Blocking nicotinic receptors with mecamylamine markedly reduced the rhythmic excitatory drive, indicating that these rhythmic inputs originate mainly from motor neurons (MNs). Inhibitory synaptic inputs persisted in the presence of the nicotinic blocker. Part of this inhibitory drive and remaining excitatory drive could be from commissural interneurons because the present study also shows that RCs receive direct crossed inhibitory and excitatory synaptic inputs. However, rhythmic synaptic inputs in RCs were also observed in hemicord preparations in the presence of mecamylamine. These results show that, during locomotor activity, RC firing properties are modulated not only by MNs but also by the ipsilateral and contralateral locomotor networks.

Key words: spinal cord; interneurons; locomotion; rhythmic; commissural interneurons; central pattern generator

\section{Introduction}

Inhibitory neurons are important elements in mammalian neuronal circuits. One of the first functionally identified groups of inhibitory neurons in the mammalian CNS were Renshaw cells (RCs) located in the ventral spinal cord (Renshaw, 1946). RCs are excited by axon collaterals from motor neurons (MNs) and provide recurrent inhibition of motor neurons (Renshaw, 1941; Eccles et al., 1954). They are one of the few identified groups of interneurons that have been shown to directly innervate MNs. Because RCs possess such unique characteristics, a large number of studies have attempted to address their functional role in motor control. A leading hypothesis is that they serve a gaincontrolling role of motor outputs (Hultborn et al., 1979, 2004; Windhorst, 1996). The firing activity of RCs during rhythmic activity such as locomotion and scratching has been studied in the cat by extracellular recordings (Feldman and Orlovsky, 1975; McCrea et al., 1980; Pratt and Jordan, 1980; Deliagina and Feldman, 1981). These studies show that RCs are rhythmically active during these motor activities. It has been suggested that RCs are

Received Dec. 2, 2005; revised April 9, 2006; accepted April 9, 2006.

This work has been supported by the Human Frontier Science Program (0.K.), the National Institutes of Health (0.K.), and the Japan Society for the Promotion of Science postdoctoral fellowship for research abroad (H.N.). We thank Y. Yanagawa for providing us with the GAD67-GFP mouse.

Correspondence should be addressed to Ole Kiehn, Department of Neuroscience, Karolinska Institutet, Retzius vag 8, 17177 Stockholm, Sweden. E-mail: o.kiehn@neuro.ki.se.

D01:10.1523/JNEUROSCI.5127-05.2006

Copyright $\odot 2006$ Society for Neuroscience $\quad$ 0270-6474/06/265320-09\$15.00/0 driven by excitatory synaptic inputs mediated by nicotinic receptors from MNs (Noga et al., 1987) and have a role in shortening the ipsilateral $\mathrm{MN}$ bursts during locomotor activity. However, because of the difficulty and the limitation of recording techniques, characterization of the synaptic modulation of RCs during locomotor activity has not been performed in detail. In the present study, we recorded activity patterns of and synaptic inputs to RCs during locomotor-like activity in the in vitro isolated spinal cord preparations from neonatal rodents that are used widely to study the neuronal mechanism underlying locomotion in mammals (for review, see Cazalets and Bertrand, 2000; Nishimaru and Kudo 2000; Schmidt and Jordan, 2000; Kiehn and Butt, 2003). The spinal neuronal networks controlling locomotion, also called central pattern generators (CPGs), remain intact in this preparation, and intracellular recordings from identified classes of interneurons can readily be performed (Butt et al., 2002). Moreover, genetically driven labeling of neurons by fluorescent proteins, such as green fluorescent protein (GFP), enabled electrophysiological recordings from visually identified neurons (Hinckley et al., 2005; Wilson et al., 2005). In this study, we took advantage of this possibility using isolated spinal cord preparations from glutamic acid decarboxylase 67 (GAD67)GFP knock-in mouse (Tamamaki et al., 2003), which enabled us to record from visually identified inhibitory interneurons localized in the ventral horn. RCs are among these GFP-positive neurons because they express GAD67 in early postnatal life (Alvarez et al., 2005). We show that RCs are rhythmically active and fire 
A
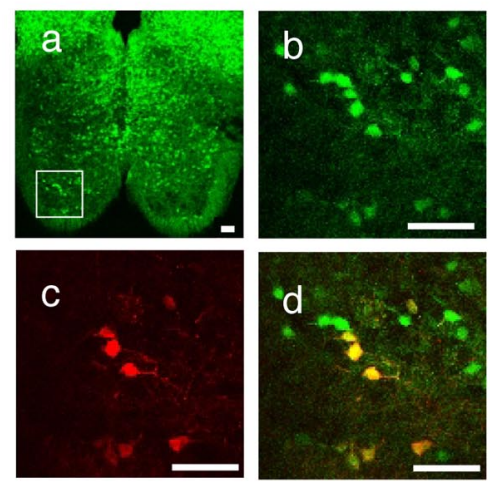

B

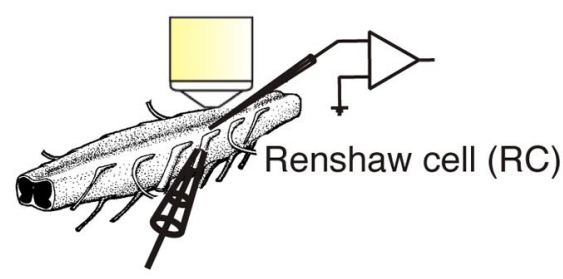

Ventral Root Stimulation (VR-S) and Recording

C

E

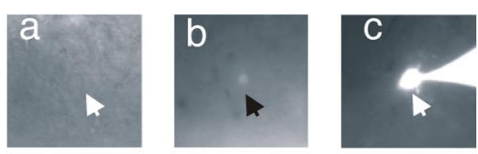

D
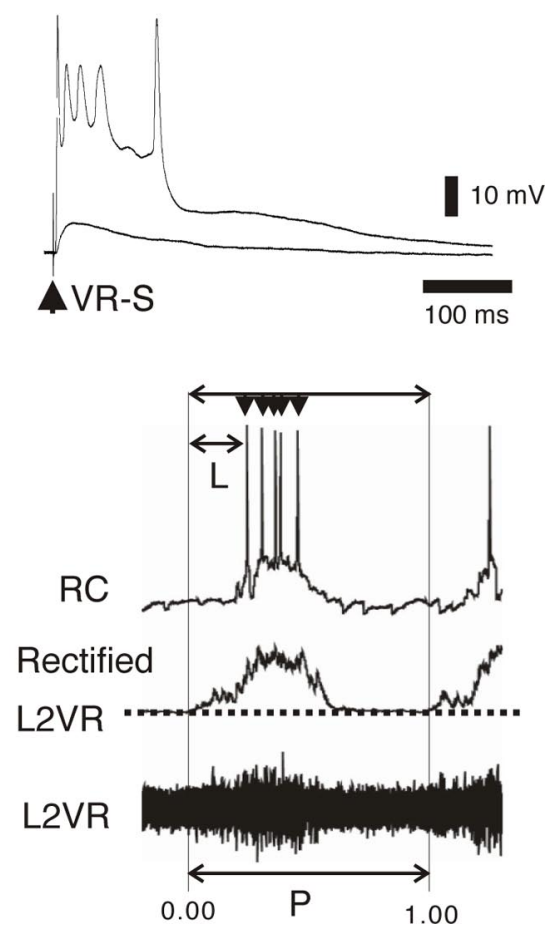

Figure 1. Identification of RCs. A, Transverse section of lumbar spinal cord showing GAD67-GFP-positive cells (a). $\boldsymbol{b}-\boldsymbol{d}$ show enlargements of the ventral cord spinal with calbindin labeling in c and superimpositions of GFP and calbindin in d. Scale bars, 50 $\mu \mathrm{m}$. $B$, Schematic of the recording setup. Suction electrode was used to stimulate and record the activity of the $L 2$ ventral root ipsilateral to the recorded RCs. C, Visually guided patching of the GFP-positive cell located in the L2 ventral horn. $\boldsymbol{a}$ and $\boldsymbol{b}$ shows an GFP-positive cell before patch-clamp recording in bright-field $(\boldsymbol{a})$ and fluorescent $(\boldsymbol{b})$ images. $\boldsymbol{c}$, The cell after filling with Alexa Fluor 488 dye in the pipette solution. $D$, Example of high-frequen cy firing of $R C s$ evoked by ventral root stimulation. Such firing was typically evoked by electrical stimulation intensity two to three times higher than the stimulus to evoke a subthreshold EPSP in the RC. $\boldsymbol{E}$, A schematic of how phase values $(\Phi)$ were calculated from the period $(P)$ and the latency $(L)$. Records from the i- $L 2$ ventral root were full-wave rectified and smoothed with low-pass filtering. For details of circular plot measurements, see Materials and Methods.

throughout either the flexor- or extensor- related motor bursts. In addition to their synaptic drive from MNs, they receive synaptic inputs from commissural interneurons with axons crossing in the midline as well as the ipsilateral locomotor network. Our results thus show that RC firing is modulated from sources other than the MNs, including inhibitory interneurons activated during the locomotor-like rhythmic activity. Their synaptic inputs and firing patterns suggest that RCs play a less pronounced role in determining the termination of motor neuron burst during locomotion than suggested previously.

\section{Materials and Methods \\ Recordings}

All procedures followed Swedish federal guidelines for animal care. In all experiments, we used heterozygote GAD67-GFP knock-in mice (Tamamaki et al., 2003) aged postnatal day $0-4$. The animals were anesthetized with isoflurane, and the spinal cords were removed as described previously (Kullander et al., 2003; Butt et al., 2005). For recording, the dissected spinal cord was placed in a chamber perfused with oxygenated Ringer's solution [in mм: $128 \mathrm{NaCl}, 4.69 \mathrm{KCl}, 25 \mathrm{NaHCO}_{3}, 1.18$ $\mathrm{KH}_{2} \mathrm{PO}_{4}, 1.25 \mathrm{MgSO}_{4}, 2.5 \mathrm{CaCl}_{2}$, and 22 glucose (aerated with $5 \% \mathrm{CO}_{2}$ in $\left.\mathrm{O}_{2}\right)$ ] at room temperature $\left(20-23^{\circ} \mathrm{C}\right)$. Electrical recordings and stimulations of the ventral roots were performed with glass suction electrodes placed in close proximity to the exit point of the ventral roots. Locomotor-like rhythmic activity was evoked by bath application of NMDA $(5-7.5 \mu \mathrm{M})$ in combination with serotonin (5-HT) $(7-10 \mu \mathrm{M})$ as described previously (Kullander et al., 2003; Butt et al., 2005). Motor neuron activity was monitored in the second lumbar segment (L2) ventral root. Like in rats (Kiehn and Kjaerulff, 1996), the major locomotor burst in L2 represents flexor activity in the mouse (Whelan et al., 2000; Bonnot et al., 2002). RCs were identified as described in Results (Nishimaru et al., 2005). Extracellular signals were amplified 10-20,000 times and bandwidth filtered at $100-1000 \mathrm{~Hz}$ with custom-designed amplifiers. Whole-cell, tight-seal recording of RCs was performed with patch electrodes pulled from thick-walled borosilicate glass (Harvard Apparatus, Holliston, $\mathrm{MA}$ ) to a final resistance of 5-8 $\mathrm{M} \Omega$ and filled with the following (in $\mathrm{mm}$ ): $138 \mathrm{~K}$-gluconate, 10 HEPES, $0.0001 \mathrm{CaCl}_{2}$, 5 ATP-Mg, and 0.3 GTP-Li. Intracellular signals were recorded with a Multiclamp 700A amplifier (Molecular Devices, Palo Alto, CA). All intracellular recordings were performed under visual guidance in an experimental setup equipped with differential interference contrast and fluorescent optics. Cells were usually recorded for $1-2 \mathrm{~h}$.

\section{Stimulation}

To activate axons from commissural interneurons, the ventral commissure in the L2 segment was electrically stimulated using a concentric bipolar stainless steel electrode (inner pole wire diameter, $12.5 \mu \mathrm{m}$; Frederick Haer Company, Bowdoinham, ME). Single pulses (200 $\mu \mathrm{s} \mathrm{du-}$ ration, $60-300 \mu \mathrm{A}$ in intensity, $5 \mathrm{~s}$ interval) were delivered to evoke responses in RCs.

\section{Data analysis}

Recorded signals were digitized $(5 \mathrm{kHz})$ and recorded to hard disk using either Clampex or Axoscope 8.2 (Molecular Devices). Off-line data analysis was performed using Axoscope 8.2, Clampfit 8.2, and Datapac 2000 (version 2.33; Run Technologies, Laguna Hills, CA). Summary statistics report the mean \pm SE unless otherwise specified.

Circular plots. Circular statistics were performed as described previously (Butt et al., 2002). Briefly, every 10th L2 burst taken from a sample of stable locomotor cycles was analyzed. The latency (Kjaerulff and Kiehn, 1996) of each RC spike was measured in relation to the duration of the ventral root burst to give the phase value $\Phi$. The mean of $10 \Phi$ was calculated using the formula outlined by Kjaerulff and Kiehn (1996). This calculation gave the preferred phase of firing (the mean vector) and the concentration of firing around the preferred phase of firing (expressed by $r$ ). A preferred phase of firing from 0 to $\sim 0.5$ means that the RC fired in-phase with the ipsilateral ventral L2 root (i-L2), whereas phase values with a direction in the range of $0.5-1.0$ approximately correspond to cells firing out-of-phase with i-L2, because cessation of i-L2 bursting occurred approximately halfway through the cycle. These data are presented in circular plots. $p$ values for the significance of $r$ were taken from Zar (1974). The preferred phase of firing shown in the circular plots is the mean vector of all of the spikes calculated from single reference analysis. We used this analysis method to examine preferred firing phase during the entire locomotor cycle in each individual RC. This analysis 
gives a simple way to summarize the firing in different cells but does not show the onset-offset timing of the firing in each cell. We therefore also used phase plots to represent the entire firing (see below, Doublereferent phase measurement).

Double-referent phase measurement. Because the ventral root bursting sometimes showed variations in duty cycle, the onset and offset values for $\mathrm{RC}$ firing with respect to the ventral root recording were calculated for all cells using double-referent measurements (Berkowitz and Stein, 1994; Stein and Daniels-McQueen, 2002). This analysis shows the onset and offset with relation to the flexor and extensor phase for each RC. Each cycle of L2 rhythmic activity was divided into bursting $(\mathrm{ON})$ phase and nonbursting (OFF) phase. We defined the start of each burst as having the phase values 0.0 and/or 1.0 and the end of each burst as having the phase value 0.5 . The latencies for RC spikes were then measured with respect to these normalized ON and OFF phases (see Fig. 3). Spikes were measured for at least 10 cycles, and bar diagrams of the duration of the mean \pm SD firing were plotted. For each cell, the mean phase in the double-reference phase plots approximately corresponded to the preferred firing phase in the circular plots if the duty cycle was 0.5 (that is, flexor and extensor phase were equally long). However, if the duty cycle was different from 0.5 , there was also difference in the preferred phase of firing (see Fig. 2) and the mean phase obtained from the double-referent phase measurement.

Current-clamp analysis. For amplitude measurements of the RC membrane oscillations during fictive locomotion, current-clamp recordings of RCs were low-pass filtered $(10 \mathrm{~Hz})$, and peak-to-trough values were measured for at least 10 cycles.

\section{Drugs}

All drugs [mecamylamine, 6-cyano-7-nitroquinoxaline-2,3-dione (CNQX), D-(-)-2-amino-5-phosphonopentanoic acid (D-AP-5), 5-HT, and NMDA] were purchased from Sigma (St. Louis, MO) or Research Biochemicals (Natick, MA). Drugs were dissolved in Ringer's solution and bath applied to the preparation.

\section{Anatomy}

The spinal cord was fixed (4\% paraformaldehyde in $0.1 \mathrm{M}$ PBS) overnight at $4^{\circ} \mathrm{C}$. Then the preparations were cryoprotected in $0.1 \mathrm{M}$ phosphate buffer with $15 \%$ sucrose and cut to $25-\mu \mathrm{m}$-thick transverse sections. To visualize calbindin in RCs, the sections were incubated with the primary antibody for calbindin 28k (rabbit polyclonal antibody; 1:5000; Swant, Bellinzona, Switzerland). Dilutions were made in $0.1 \mathrm{M}$ PBS with $0.1 \%$ Triton $\mathrm{X}-100, \mathrm{pH} 7.4$, and incubations of the primary antibody mixture lasted for $48 \mathrm{~h}$ at $4^{\circ} \mathrm{C}$.

\section{Results}

\section{Identification of RCs}

RCs, like most other inhibitory spinal neurons, express GABA during embryonic and early postnatal life. They are among the GFP-positive cells that are located in the most ventral part of the ventral horn (Fig. $1 A$ ). RCs also show specific calbindin $28 \mathrm{k}$ immunoreactivity (Carr et al., 1998), as shown in Figure 1, $A c$ and $A d$. GFP-positive cells in the ventral cord were visually patched in the L2 (Fig. $1 B, C$ ) and were identified as RCs by electrically stimulating the L2 ventral root to evoke short-latency EPSPs, which were mostly blocked by bath application of the nicotinic receptor antagonists mecamylamine or tubocurarine (Mentis et al., 2005; Nishimaru et al., 2005). Some cells were also filled with Alexa Fluor dyes to confirm their expression of calbindin $28 \mathrm{k}$ after recording (Nishimaru et al., 2005). We also observed highfrequency bursting after ventral root stimulation (Fig. 1D), a hallmark of RCs as demonstrated in previous studies from both adult and immature animals (Renshaw, 1946; Eccles et al., 1954; Naka, 1964; Mentis et al., 2005).

\section{Rhythmicity of RCs during locomotion}

The firing behavior of RCs during locomotor activity was examined in 22 identified RCs during locomotor-like rhythmic activity
A

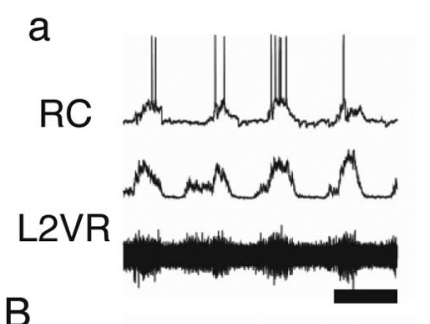

b
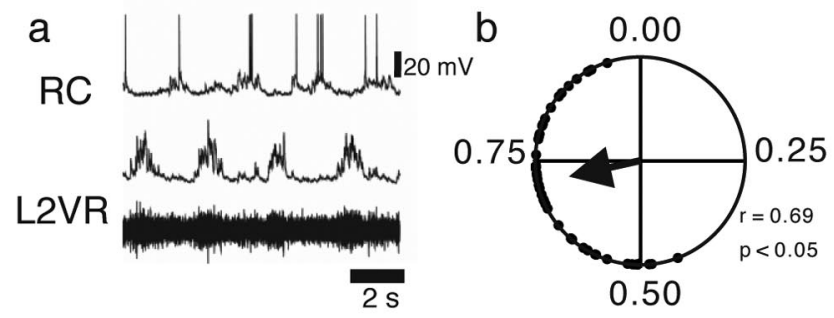

C

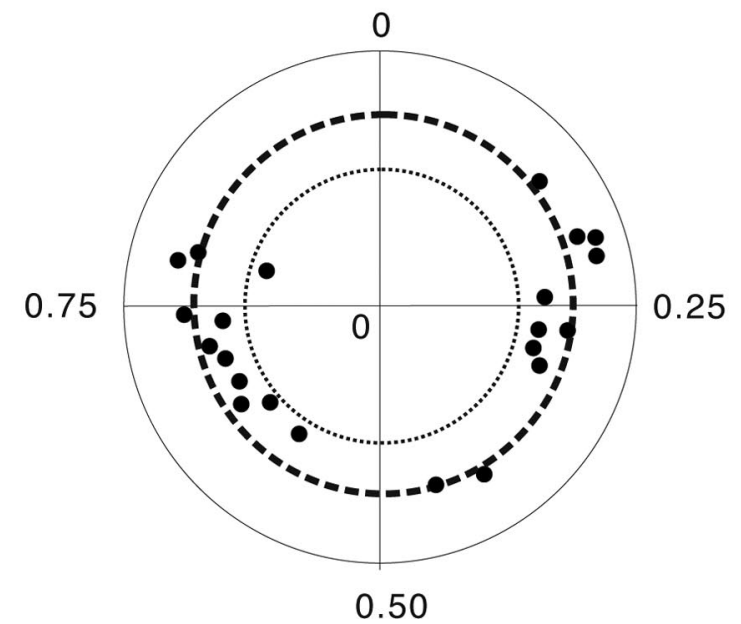

Figure 2. Firing patterns of $\mathrm{RCS}$ during locomotor-like activity. $A, B$, Examples of $\mathrm{RC}$ firing and their circular plot showing their preferred firing phase. The top traces of $\boldsymbol{A} \boldsymbol{a}$ and $\boldsymbol{B} \boldsymbol{a}$ shows $\mathrm{RC}$ firing $(\mathrm{RC})$, and the two bottom traces shows the raw (bottom) and rectified (middle) recordings of L2 ventral root (L2VR). Circular plots for these RCs are shown in $\boldsymbol{A} \boldsymbol{b}$ and $\boldsymbol{B} \boldsymbol{b}$. Both cells were significantly rhythmic. Vertical calibration bar indicates the amplitude of the $\mathrm{RC}$ recordings. C, Circular phase diagram showing preferred phase of firing and the significance level of the phase relationship for all recorded RCs. The outer dotted circle indicates high significance $(p<0.001)$, and the inner dotted circle indicates significance $(0.05<p<0.001)$. The dots inside the inner dotted circle are nonsignificant.

induced by bath application of 5-HT and NMDA. The locomotor activity was monitored in the L2 ipsilateral to the side of the whole-cell recording. The main bursts of L2 during locomotor activity in rodents represent flexor MN activity (Kiehn and Kjaerulff, 1996; Whelan et al., 2000; Bonnot et al., 2002). Typically, RCs fired few spikes (one to eight) at relatively low frequencies $(<20 \mathrm{~Hz})$. Examples of this firing pattern are shown for two RCs in Figure 2, $A a$ and $B a$. A quantitative measure of the firing behavior is the preferred phase of firing (Fig. $1 E$ ) (for details of the analysis, see Materials and Methods). Figure 2, $A b$ and $B b$, shows the preferred phases of firing for the cells in $A a$ and $B a$, respectively. The preferred phase of firing of all of the recorded RCs that were significantly rhythmic (21 of 22) during locomotor-like activity is plotted in Figure $2 C$ in which the i-L2 bursting activity is approximately spanning phases from 0.0 to 0.5 .

Fifty percent of the recorded RCs ( 11 of 22 cells) fired in-phase 


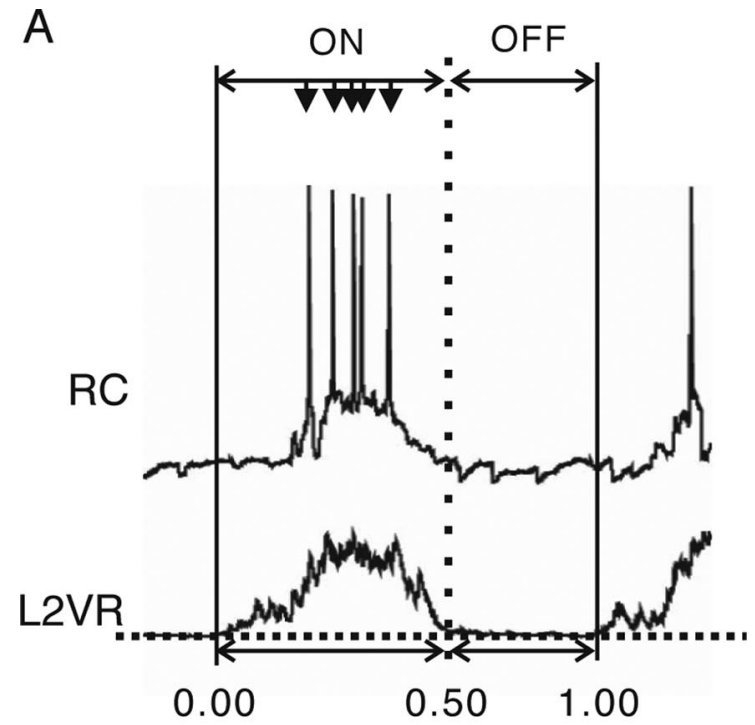

B

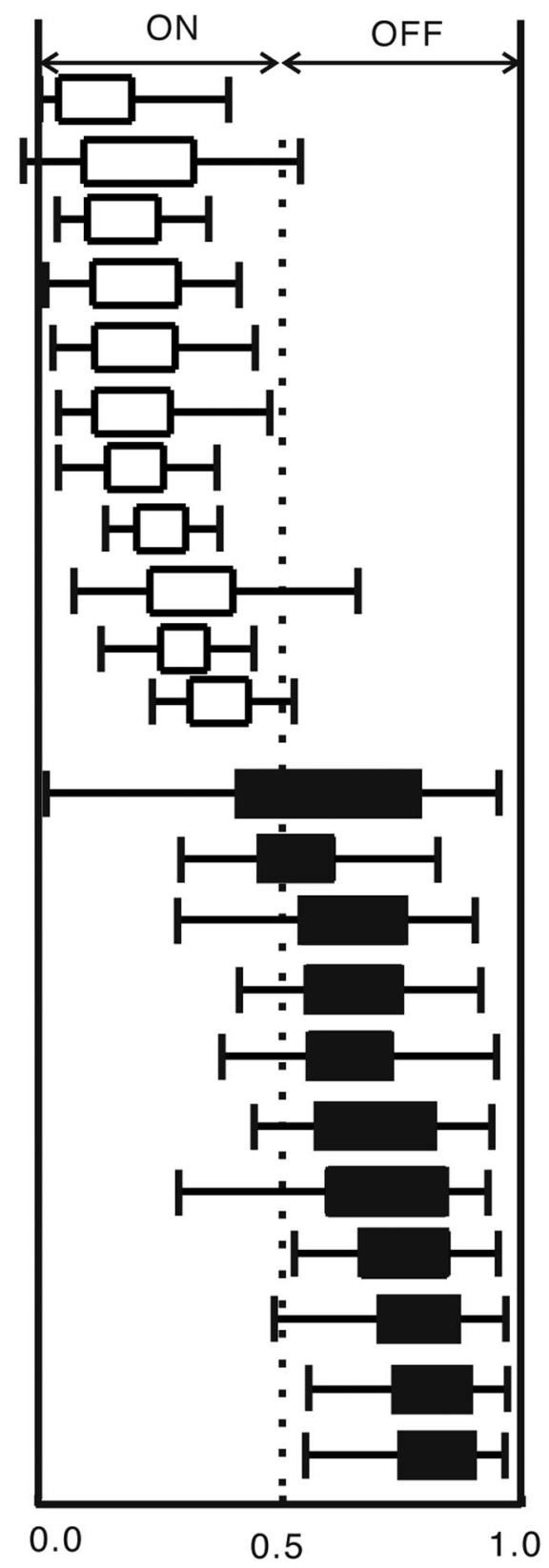

with the i-L2 burst (Fig. 2C), whereas the remaining RCs fired out-of-phase with i-L2 activity (Fig. $2 B$ ). This observation suggests that we recorded from both flexor- and extensor-related RCs. The preferred phase of firing within these two categories were, however, dispersed with most cells having a preferred phase of firing $\sim 0.25$ (midflexor) or 0.75 (midextensor). Thus, this analysis did not confirm previous findings in the cat spinal cord, suggesting that RCs tend to fire at the end of the flexor or extensor motor burst (Noga et al., 1987). Because the preferred phase of firing does not denote onset and offset timings, we also produced phase diagrams for all cells. To accurately relate the RC firing to the flexor $(\mathrm{ON})$ and extensor (OFF) phase, we used doublereferent measurements (see Methods and Materials) (Fig. 3A). Like the circular measurements, we found with this analysis that RCs fired throughout the flexor and extensor phase (Fig. 3B), although it also showed that some cells did tend to fire in the late offset phase. These results show that RCs are active throughout the locomotor phase, with each cell firing either during the flexor or extensor phase, indicating that each RC is coupled with a functionally synonymous $\mathrm{MN}$ group during locomotion in the mouse spinal cord.

\section{Rhythmic synaptic inputs to RCs}

We next asked what is the nature of the synaptic drive underlying $\mathrm{RC}$ firing during the locomotor activity. When rhythmically active RCs were voltage clamped close to the resting potential ( -60 $\mathrm{mV}$ ) of the cell, a barrage of inward currents, presumably EPSCs, were observed in the same phase as the preferred phase of firing observed in current clamp (Fig. $4 A-C$ ). This result shows that rhythmic firing of the RCs is mainly attributable to phasic excitatory synaptic inputs. When the membrane potential was depolarized to $-40 \mathrm{mV}$, which is above the chloride ion reversal potential (approximately $-70 \mathrm{mV}$ in the present experimental condition), the picture was more complex: the inward currents were smaller, and outward currents, presumably IPSCs, became prominent. Below, we will consider the details of these excitatory and inhibitory synaptic inputs separately.

\section{Rhythmic synaptic inputs from MNs}

Because MNs have excitatory collaterals onto RCs, we first asked whether the main component of the excitatory drive originates from MNs. We tested this hypothesis by using the nicotinic receptor blocker mecamylamine $(100 \mu \mathrm{M} ; n=6)$ to block the action of acetylcholine released from motor neurons (Curtis and Ryall, 1966; Mentis et al., 2005; Nishimaru et al., 2005). The rhythmic voltage oscillations (Fig. 4A) and the rhythmic EPSCs observed during locomotion were noticeably reduced in amplitude and frequency by mecamylamine at both $-60 \mathrm{mV}$ (Fig. $4 \mathrm{Bb}$ ) and $-40 \mathrm{mV}$ (Fig. $4 \mathrm{Cb}$ ), making the rhythmic inhibition more clear. In current clamp, the trough-to-peak amplitude of the depolarization was reduced to $57.9 \pm 7.2 \%$ of the control after bath application of mecamylamine. This reduction in the trough-to-peak amplitude of the depolarization led to reduced or

Figure 3. Double-referent phase measurement of the RC rhythmic firing. A, Schematic of technique used to measure double-referent phase of $\mathrm{RC}$ activity (top trace) during locomotorlike activity with respect to the i-L2 activity (L2VR; bottom trace). The latencies for RC spikes (arrowheads) were measured with respect to the normalized ON and OFF phases of i-L2. For additional details, see Materials and Methods. $\boldsymbol{B}$, Bar diagrams of the duration of the mean \pm $S D$ firing of all recorded RCs. RCs that fired preferably in the flexor (ON) phase are indicated by open bars $(n=11)$, whereas those fired in the extensor (OFF) are indicated by filled bars ( $n=$ 11). 
abolished firing and is similar to the reduction mecamylamine has on ventral root-evoked excitatory responses recorded in RCs (Mentis et al., 2005; Nishimaru et al., 2005).

In accordance with previous observations (Mentis et al., 2005), there was little or no recovery after wash of mecamylamine (data not shown). The changes in the excitatory drive are not likely to be caused by major changes in overall locomotor drive because, although the locomotor period in the population of preparations became significantly longer with bath application of mecamylamine $(2.31 \pm 0.04 \mathrm{~s}$ before and $2.48 \pm 0.04 \mathrm{~s}$ after), the absolute changes in locomotor periods were small (Fig. 4). Together, our results indicate that cholinergic synaptic inputs most likely deriving from MNs are the main source for the excitatory drive of RCs during locomotion.

\section{Inhibitory synaptic currents in RCs}

The IPSCs that persisted in the presence of the nicotinic antagonist were seen in all RCs $(n=22)$. Six of the RCs showed pronounced IPSCs in both the nonfiring phase and firing phase of the cell (Figs. $4 C a, 5 A a, A b)$, suggesting that some RCs receive a push-pull drive consisting of excitatory synaptic inputs alternating with inhibitory synaptic inputs during locomotor-like rhythmic activity similar to what has been observed in rodent MNs and other rodent interneurons (Hochman and Schmidt, 1998; Raastad and Kiehn, 2000; Butt et al., 2002). In the remaining $\mathrm{RCs}$, the inhibition that appeared in-phase with the RC firing was more pronounced than in the inactive phase. The inhibition that appeared in-phase with the RC firing was sometimes strong enough to pause the firing (Fig. $5 B, C$ ). Both the in-phase and out-of-phase inhibition was blocked by bath application of a combination of strychnine $(0.5 \mu \mathrm{M})$ and bicuculline $(3 \mu \mathrm{M})$ (Fig. 5Ac) $(n=4)$. In the following and in Discussion, we address the possible source of the inhibition.

\section{Commissural interneurons provide synaptic inputs to RCs}

It has been shown in previous studies that commissural interneurons make both excitatory and inhibitory connections across the midline in mammals (Butt and Kiehn 2003; Hammar et al., 2004). The remaining rhythmic inhibition and excitation may therefore originate from cross-cord actions onto RCs. To test whether commissural interneurons have synaptic inputs to RCs, the ventral commissure was electrically stimulated with a bipolar stimulation electrode. Short-latency (5-12 ms) IPSCs that were blocked by a mixture of bicuculline and strychnine were evoked in six of nine RCs (Fig. 6Aa). Midline stimulation evoked shortlatency excitatory inputs blocked by CNQX $(20 \mu \mathrm{M})$ and AP-5 $(20 \mu \mathrm{M})$ in three RCs (Fig. 6Ab). Two of three RCs that received short-latency EPSCs also received IPSPs (Fig. $6 \mathrm{Ba}$ ), which be- came prominent when the membrane potential was clamped to $-40 \mathrm{mV}$ (Fig. 6 Bb). Because these long-latency IPSCs were also blocked by CNQX $(20 \mu \mathrm{M})$ and AP-5 $(20 \mu \mathrm{M})$ (for example, see Fig. $6 \mathrm{Ab}$ ) they are likely to be mediated by polysynaptic pathways involving glutamatergic commissural interneurons (Kjaerulff and Kiehn, 1997; Butt and Kiehn, 2003). These results show that RCs receive excitatory and inhibitory commissural inputs that may provide rhythmic excitatory and inhibitory drive during locomotion across the cord. To remove such commissural influence on RCs firing, we recorded from RCs in hemicords, that is in spinal cords that were split longitudinally along the midline ( $n=$ 4). As shown in Figure 6, $\mathrm{Ca}$ and $\mathrm{Da}$, excitatory and inhibitory rhythmic inputs to RCs similar to those recorded in the intact spinal cord could be observed. Moreover, inhibitory synaptic inputs persisted in the presence of mecamylamine, which reduced the excitatory synaptic inputs (Fig. $6 C b, D b$ ). These results indicate that, although rhythmic crossed inputs might influence RC activity, ipsilateral locomotor networks primarily contribute to the firing pattern of the RCs through excitation and inhibition. 
A

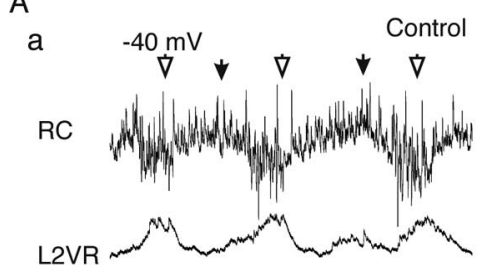

b +MEC

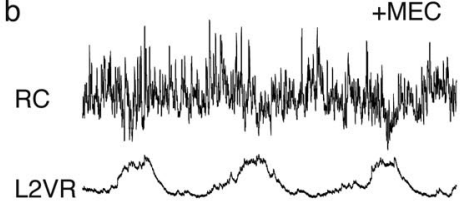

C

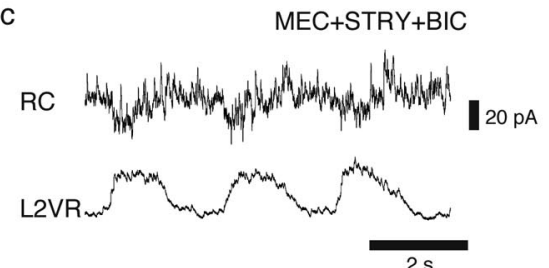

B

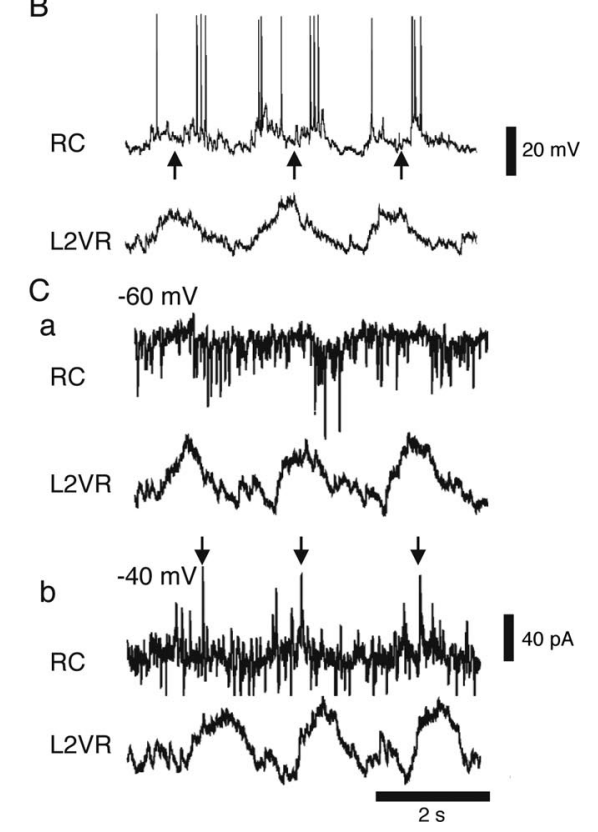

Figure 5. Inhibitory synaptic inputs during rhythmic motor activity in RCs. $\boldsymbol{A}$, IPSCs in-phase (open arrowhead) and out-ofphase (filled arrowhead) with rhythmic EPSCs observed in control ( $\boldsymbol{a}$ ) and in the presence of mecamylamine $(\boldsymbol{b})$ were blocked by bath application of strychnine $(0.5 \mu \mathrm{m})$ and bicuculline $(3 \mu \mathrm{m} ; \mathrm{c})$. The membrane potential of the cell was held at $-40 \mathrm{mV}$. Bottom trace shows the rectified $L 2 V R$ recording. $\boldsymbol{B}$, Example of an $\mathrm{RC}$ inhibited at the peak of the $\mathrm{i}-\mathrm{L} 2$ burst (L2VR; arrows). $\boldsymbol{C}$, Voltageclamp recordings $(-60 \mathrm{mV}, \boldsymbol{a} ;-40 \mathrm{mV}, \boldsymbol{b})$ of the same cell as shown in $\boldsymbol{B}$. Arrows indicate the large phasic IPSCs coinciding with the peak of firing in current clamp. Vertical calibration bars indicate the amplitude of the $\mathrm{RC}$ recordings

\section{Discussion}

This study describes the first intracellular analysis of RCs firing properties during rhythmic motor activity in mammals. We used the GAD67-GFP knock-in mouse to facilitate the identification of RCs in isolated spinal cord preparations taken from neonatal mice. The synaptic inputs to RCs, with mixed excitation and inhibition, are more complex than hitherto appreciated from extracellular recordings. Almost all of the RCs were strongly modulated during locomotor activity and fired throughout the locomotor cycle. They received strong nicotinic excitatory synaptic drive most likely derived from MNs. Moreover, we have shown that RCs receive synaptic inputs from commissural interneurons and from ipsilateral locomotor networks.

\section{Locomotor-related firing patterns of RCs}

Electrical activity of RCs during fictive locomotion has been examined previously in the cat spinal cord (McCrea et al., 1980; Pratt and Jordan, 1980; Noga et al., 1987). In these studies, it was shown that RCs fired rhythmically in bursts of spikes with activity in one phase of the step cycle and silence in the opposite phase. A similar firing pattern was also found in the present study, indicating that such firing properties of RCs are shared in mammalian quadrupeds. In the present study, we did not directly relate the RCs to specific MN pools but classified them based on their preferred phase of firing: in-phase or out-of-phase with ipsilateral L2 activity as either flexor- or extensor-related RCs, respectively, because the main burst activity in L2 during drug-induced locomotor-like activity in rodents represents flexor activity (Kiehn and Kjaerulff, 1996; Whelan et al., 2000; Bonnot et al., 2002). However, it has been shown that the L2 segment also contains $\mathrm{MN}$ innervating extensor muscles in rats (Nicolopoulos-Stournaras and Iles, 1983) and mouse (McHanwell and Biscoe, 1981). Our results therefore indicate that RCs are functionally coupled with both flexor and extensor MNs during locomotor activity in the neonatal mouse spinal cord as it has been shown in the cat.

In the cat spinal cord, it has been shown that the maximal firing activity in RCs occurs at the end of the burst of related MNs (McCrea et al., 1980; Pratt and Jordan, 1980). In the present study, we found this type of firing also in some RCs (Fig. 3). However, the population of flexor- and extensor-related RCs fired throughout the flexor and extensor phases, respectively (Fig. 3), with a concentration of preferred phases of firing in the middle of the corresponding flexor and extensor phases (Fig. 2). Interestingly, in some cells, we observed phasic inhibition that coincides with the peak of firing in the corresponding $\mathrm{MN}$ burst. This particular pattern of inhibitory input, which paused the RC firing, indicates that RCs are actively inhibited during rhythmic activity to reduce their firing. Altogether, the firing pattern reported here is more dynamic and diverse than has been reported previously for RC rhythmic firing in mammals.

\section{Synaptic inputs to RCs}

The present study shows that RCs receive both excitatory and inhibitory synaptic inputs during the locomotor-like rhythmic activity. The possible sources of these synaptic inputs are summarized in Figure 7.

The major synaptic inputs to RCs during locomotion are excitatory. These excitatory synaptic inputs are reduced in amplitude by blocking nicotinic receptors, indicating that they are mostly likely coming from motor neuron collaterals. This observation is in agreement with a previous study in the cat in which rhythmic firing during fictive locomotion was reduced or abolished after administrating mecamylamine in $\sim 80 \%$ of the extracellularly recorded RCs (Noga et al., 1987). The most likely candidate for the receptors mediating the remaining EPSCs that were observed in the presence of mecamylamine are ionotropic glutamate receptors whose activation is essential for locomotoractivity in the rodent spinal cord (Bracci et al., 1998; Nishimaru et al., 2000; Whelan et al., 2000). Although we observed direct glutamatergic excitation of RCs from the contralateral side, mecamylamine-resistant EPSCs were also recorded in hemicords. The source of these EPSCs is therefore more likely to be either ipsilaterally projecting excitatory interneurons in the locomotor network or MNs, which have been shown recently to release glutamate as well as acetylcholine onto RCs (Mentis et al., 2005; Nishimaru et al., 2005). As for the former possibility, two populations of excitatory, ipsilaterally projecting, putative CPG interneurons have been identified recently: interneurons that express the transcription factor HB9 (Hinckley et al., 2005; Wilson et al., 2005) and a subset of neurons expressing the axon guidance marker EphA4 (Butt et al., 2005). Both types of neurons fire during the peak of the ipsilateral ventral root burst, ideal for providing direct or indirect excitation of RCs. As for the latter possibility, glutamate has been shown recently to be responsible for $\sim 20-30 \%$ of the amplitude of the ventral root-evoked EPSCs in RCs (Mentis et al., 2005; Nishimaru et al., 2005). However, 
because there is presently no way of blocking the glutamatergic input selectively from MNs or interneurons, we cannot more specifically determine the source of the remaining rhythmic EPSCs.

In the present study, we found that RCs receive rhythmic glycinergic and/or GABAergic IPSCs in both active and nonactive phases during locomotion showing the possibility that RC firing is actively suppressed during locomotion as suggested previously (Severin et al., 1968; Bergmans et al., 1969). Such inhibition might be functioning to relieve MNs from recurrent inhibition during locomotion. There are two possibilities for the origin of the inhibition: the inhibitory interneurons in the locomotor network or other RCs. Although we cannot completely rule out the latter case, we are in favor of the former explanation. It has been shown that RC inhibition is mainly between RCs acting on antagonist MN pools (Ryall, 1970; Windhorst, 1996). This RC connectivity could therefore explain the inhibition in the nonactive phase but not the inhibition in the active phase. Moreover, the IPSC frequency and amplitude in the active phase changed little after application of mecamylamine, indicating that this rhythmic inhibition is less dependent on $\mathrm{MN}$ firing, which would be the case if RCs were involved. Therefore, the most likely explanation is that RCs receive inhibition in their active phase directly from other interneurons in the locomotor network. The source of this inhibition is likely to be from the ipsilaterally located CPG because we showed that inhibition in the active phase was also present in hemicord preparations. The inhibition arriving in the nonactive phase was generally small but provided a timing signal to the RCs. Because this inhibition also persisted in the hemicord, it could have originated from other RCs (see above) or from CPG neurons. Because the amplitude and frequency of these IPSCs remain unchanged after mecamylamine, we favor the latter explanation.

In the present study, we also showed that RCs receive direct excitatory and inhibitory commissural inputs. The exact functional role of this input during locomotion is unclear at present. However, it is noteworthy that the excitatory drive to RCs could serve as a coordinating signal for muscle synergies across the spinal cord through polysynaptic inhibitory actions onto contralateral MNs (Butt and Kiehn, 2003). The present analysis of the commissural interneuron influence onto RCs is, however, hampered by the fact that we were not able to simultaneously record from commissural interneurons and monitor recordings.
A

a

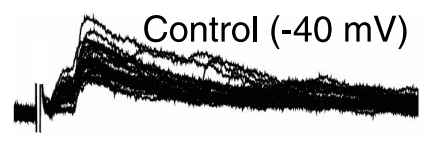

STRY+BIC (-40 mV)

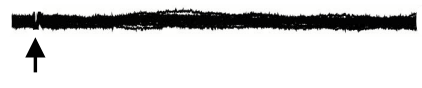

B

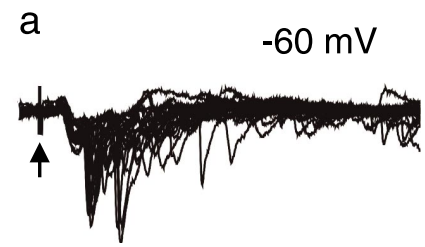

C a

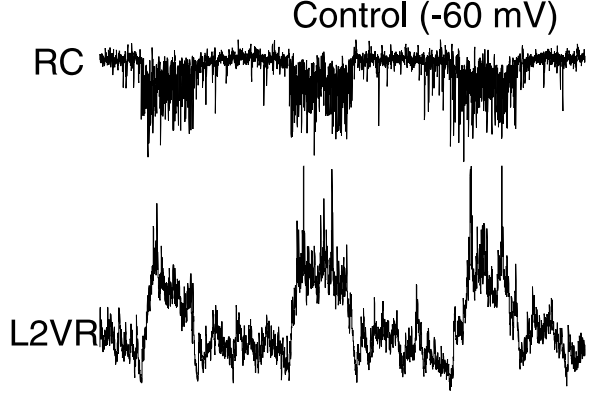

D a<smiles>CCOC(=O)OCC</smiles>

$\mathrm{RC}$
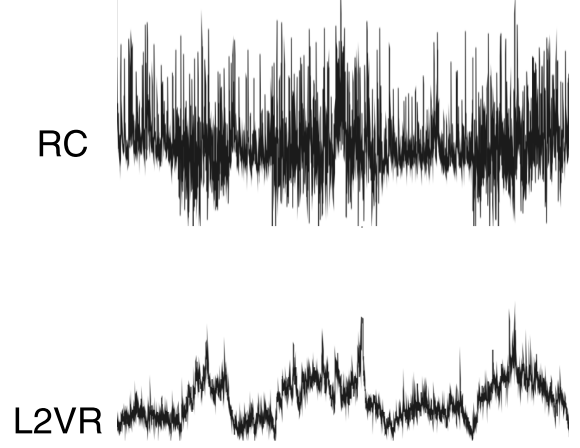

b
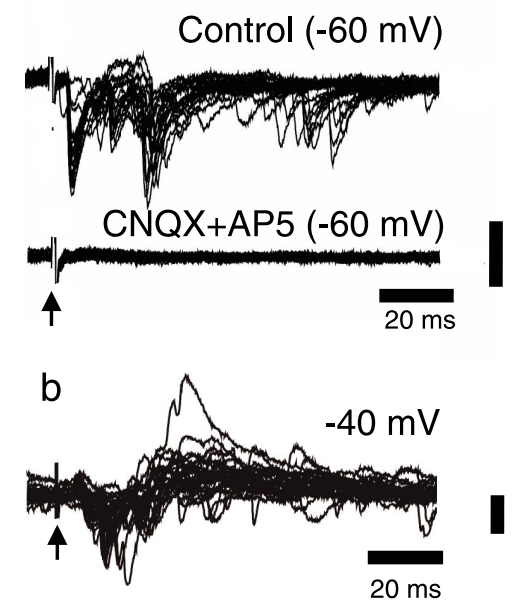

b

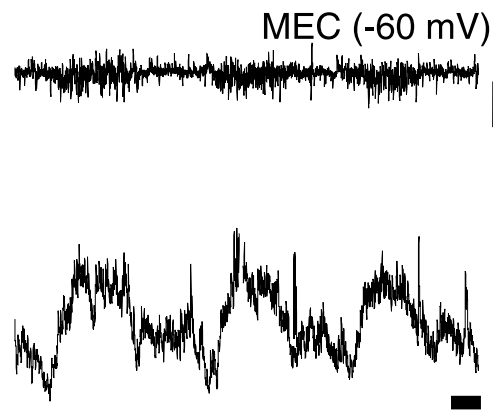

b

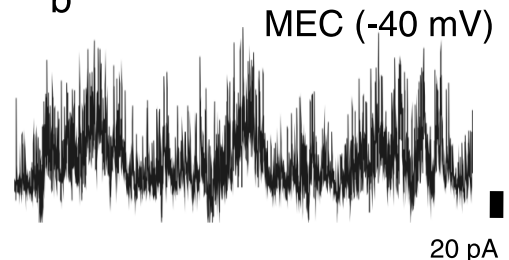

$20 \mathrm{pA}$

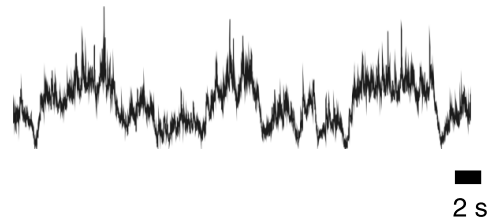

Figure 6. Contralateral and ipsilateral inputs to RCS. $\boldsymbol{A}$, Examples of IPSCS $(\boldsymbol{a})$ and $\operatorname{EPSCs}(\boldsymbol{b})$ evoked by electrical stimulation of the contralateral side. The two recordings are from different cells. IPSCS were blocked by blocking glycine and GABA $A_{A}$ receptors $($ STRY + BIC, strychnine plus bicuculline; bottom trace, $\boldsymbol{a})$, whereas EPSCs were abolished by NMDA (AP-5; $20 \mu \mathrm{M})$ and non-NMDA receptor antagonists ( $C N Q X, 20 \mu \mathrm{m}$; bottom trace, $\boldsymbol{b})$. $\boldsymbol{B}$, An example of mixed EPSCs and IPSCs evoked by contralateral stimulation. All traces in $\boldsymbol{A}$ and $\boldsymbol{B}$ are of 20 superimposed traces, and the arrowheads indicate the timing of the stimulus. $\boldsymbol{C}$, Rhythmic synaptic activity in RCs in the hemicord preparation (a). Similar to the intact cord preparation, mecamylamine (MEC) markedly reduced the rhythmic EPSCS (b). D, IPSCs observed when the membrane potential was clamped to $-40 \mathrm{mV}$ in an $\mathrm{RC}$ recorded in a hemicord preparation $(\boldsymbol{a})$. The IPSCs were resistant to mecamylamine $(\boldsymbol{b})$. Vertical calibration bars indicate the amplitude of the RC

their rhythmic activity. Moreover, we cannot completely rule out the possibility of current spread from the stimulating electrode to ipsilaterally located neurons, although we used a bipolar stimulation electrode to minimize this problem. Furthermore, it has 


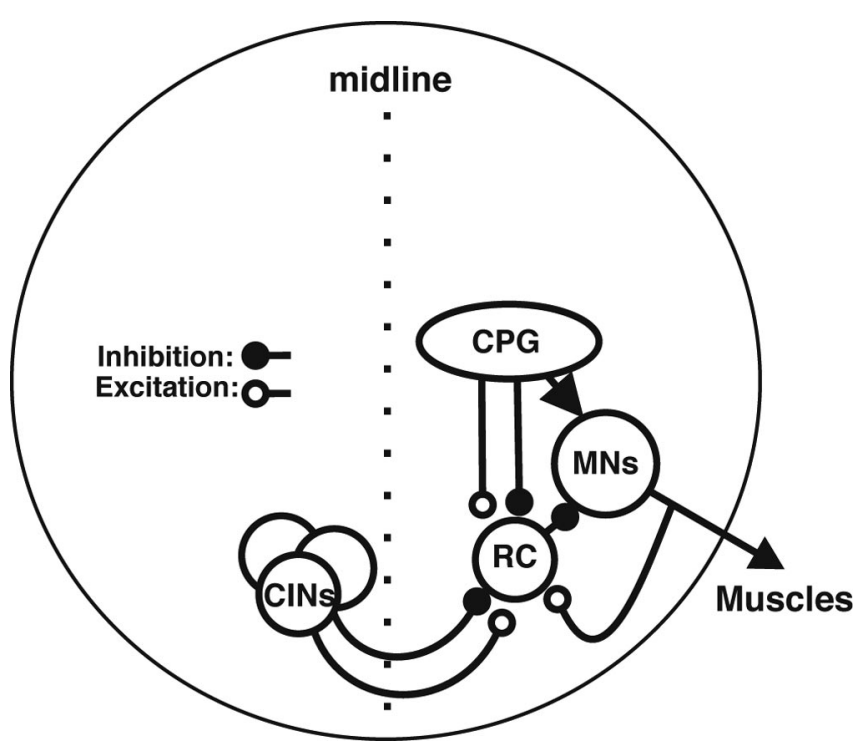

Figure 7. A schematic drawing of possible synaptic inputs to RCs during locomotor rhythm generation. Excitatory synaptic inputs are represented by open circles, and inhibitory connections are represented by filled circles. CIN, Commissural interneurons.

been shown that some commissural interneurons have ipsilateral collaterals (Petko et al., 2004). Such axons could be retrogradely stimulated, resulting in recording the ipsilateral effects of commissural interneurons in RCs. Altogether, more studies are needed to clarify the commissural neuron-RC interaction during locomotion (Jankowska et al., 2005).

In conclusion, we described the nature of the firing and synaptic inputs to identified RCs during locomotor activity in rodents by using isolated spinal cord preparations taken from GAD67-GFP knock-in mice. RCs fire throughout the ipsilateral ventral root burst. The rhythmic synaptic input to RCs is complex, with mixed excitation and inhibition in the bursting phase alternating with rhythmic inhibition in the nonbursting phase originating from ipsilateral locomotor networks and/or MNs and synaptic inputs from commissural interneurons. The quenching of firing in the bursting phase and the general firing pattern through the active motor neuron phase suggest that RCs do not play a determining role in motor neuron burst termination during locomotion.

\section{References}

Alvarez FJ, Jonas PC, Sapir T, Hartley R, Berrocal MC, Geiman EJ, Todd AJ, Goulding M (2005) Postnatal phenotype and localization of spinal cord V1 derived interneurons. J Comp Neurol 493:177-192.

Bergmans J, Burke R, Lundberg A (1969) Inhibition of transmission in the recurrent inhibitory pathway to motoneurones. Brain Res 13:600-602.

Berkowitz A, Stein PS (1994) Activity of descending propriospinal axons in the turtle hindlimb enlargement during two forms of fictive scratching: phase analyses. J Neurosci 14:5105-5119.

Bonnot A, Whelan PJ, Mentis GZ, O'Donovan MJ (2002) Spatiotemporal pattern of motoneuron activation in the rostral lumbar and the sacral segments during locomotor-like activity in the neonatal mouse spinal cord. J Neurosci 22:RC203(1-6).

Bracci E, Beato M, Nistri A (1998) Extracellular $\mathrm{K}^{+}$induces locomotor-like patterns in the rat spinal cord in vitro: comparison with NMDA or 5-HT induced activity. J Neurophysiol 79:2643-2652.

Butt SJ, Kiehn O (2003) Functional identification of interneurons responsible for left-right coordination of hindlimbs in mammals. Neuron 38:953-963.

Butt SJ, Harris-Warrick RM, Kiehn O (2002) Firing properties of identified interneuron populations in the mammalian hindlimb central pattern generator. J Neurosci 22:9961-9971.
Butt SJ, Lundfald L, Kiehn O (2005) EphA4 defines a class of excitatory locomotor-related interneurons. Proc Natl Acad Sci USA 102:14098-14103.

Carr PA, Alvarez FJ, Leman EA, Fyffe RE (1998) Calbindin D28k expression in immunohistochemically identified Renshaw cells. NeuroReport 9:2657-2661.

Cazalets JR, Bertrand S (2000) Ubiquity of motor networks in the spinal cord of vertebrates. Brain Res Bull 53:627-634.

Curtis DR, Ryall RW (1966) The acetylcholine receptors of Renshaw cells. Exp Brain Res 1966 2:66-80.

Deliagina TG, Feldman AG (1981) Activity of Renshaw cells during fictive scratch reflex in the cat. Exp Brain Res 42:108-115.

Eccles JC, Fatt P, Koketsu K (1954) Cholinergic and inhibitory synapses in a pathway from motor-axon collaterals to motoneurones. J Physiol (Lond) 126:524-562.

Feldman AG, Orlovsky GN (1975) Activity of interneurons mediating reciprocal 1a inhibition during locomotion. Brain Res 84:181-194.

Hammar I, Bannatyne BA, Maxwell DJ, Edgley SA, Jankowska E (2004) The actions of monoamines and distribution of noradrenergic and serotoninergic contacts on different subpopulations of commissural interneurons in the cat spinal cord. Eur J Neurosci 19:1305-1316.

Hinckley CA, Hartley R, Wu L, Todd A, Ziskind-Conhaim L (2005) Locomotor-like rhythms in a genetically distinct cluster of interneurons in the mammalian spinal cord. J Neurophysiol 93:1439-1449.

Hochman S, Schmidt BJ (1998) Whole cell recordings of lumbar motoneurons during locomotor-like activity in the in vitro neonatal rat spinal cord. J Neurophysiol 79:743-752.

Hultborn H, Lindstrom S, Wigstrom H (1979) On the function of recurrent inhibition in the spinal cord. Exp Brain Res 37:399-403.

Hultborn H, Brownstone RB, Toth TI, Gossard JP (2004) Key mechanisms for setting the input-output gain across the motoneuron pool. Prog Brain Res 143:77-95.

Jankowska E, Krutki P, Matsuyama K (2005) Relative contribution of Ia inhibitory interneurones to inhibition of feline contralateral motoneurones evoked via commissural interneurones. J Physiol (Lond) 568:617-628.

Kiehn O, Butt SJ (2003) Physiological, anatomical and genetic identification of CPG neurons in the developing mammalian spinal cord. Prog Neurobiol 70:347-361.

Kiehn O, Kjaerulff O (1996) Spatiotemporal characteristics of 5-HT and dopamine-induced rhythmic hindlimb activity in the in vitro neonatal rat. J Neurophysiol 75:1472-1482.

Kjaerulff O, Kiehn O (1996) Distribution of networks generating and coordinating locomotor activity in the neonatal rat spinal cord in vitro: a lesion study. J Neurosci 16:5777-5794.

Kjaerulff O, Kiehn O (1997) Crossed rhythmic synaptic input to motoneurons during selective activation of the contralateral spinal locomotor network. J Neurosci 17:9433-9447.

Kullander K, Butt SJ, Lebret JM, Lundfald L, Restrepo CE, Rydstrom A, Klein R, Kiehn O (2003) Role of EphA4 and EphrinB3 in local neuronal circuits that control walking. Science 299:1889-1892.

McCrea DA, Pratt CA, Jordan LM (1980) Renshaw cell activity and recurrent effects on motoneurons during fictive locomotion. J Neurophysiol 44:475-488.

McHanwell S, Biscoe TJ (1981) The localization of motoneurons supplying the hindlimb muscles of the mouse. Philos Trans R Soc Lond B Biol Sci 293:477-508.

Mentis GZ, Alvarez FJ, Bonnot A, Richards DS, Gonzalez-Forero D, Zerda R, O'Donovan MJ (2005) Noncholinergic excitatory actions of motoneurons in the neonatal mammalian spinal cord. Proc Natl Acad Sci USA 102:7344-7349.

Naka KI (1964) Electrophysiology of the fetal spinal cord. II. Interaction among peripheral inputs and recurrent inhibition. J Gen Physiol 47:1023-1038.

Nicolopoulos-Stournaras S, Iles JF (1983) Motor neuron columns in the lumbar spinal cord of the rat. J Comp Neurol 217:75-85.

Nishimaru H, Kudo N (2000) Formation of the central pattern generator for locomotion in the rat and mouse. Brain Res Bull 53:661-669.

Nishimaru H, Takizawa H, Kudo N (2000) 5-Hydroxytryptamine-induced locomotor rhythm in the neonatal mouse spinal cord in vitro. Neurosci Lett 280:187-190.

Nishimaru H, Restrepo CE, Ryge J, Yanagawa Y, Kiehn O (2005) Mamma- 
lian motor neurons corelease glutamate and acetylcholine at central synapses. Proc Natl Acad Sci USA 102:5245-5249.

Noga BR, Shefchyk SJ, Jamal J, Jordan LM (1987) The role of Renshaw cells in locomotion: antagonism of their excitation from motor axon collaterals with intravenous mecamylamine. Exp Brain Res 66:99-105.

Petko M, Veress G, Vereb G, Storm-Mathisen J, Antal M (2004) Commissural propriospinal connections between the lateral aspects of laminae III-IV in the lumbar spinal cord of rats. J Comp Neurol 480:364-377.

Pratt CA, Jordan LM (1980) Recurrent inhibition of motoneurons in decerebrate cats during controlled treadmill locomotion. J Neurophysiol 44:489-500.

Raastad M, Kiehn O (2000) Spike coding during locomotor network activity in ventrally located neurons in the isolated spinal cord from neonatal rat. J Neurophysiol 83:2825-2834.

Renshaw B (1941) Influence of discharge of motoneurons upon excitation of neighboring motoneurons. J Neurophysiol 4:167-183.

Renshaw B (1946) Central effects of centripetal impulses in axons of spinal ventral roots. J Neurophysiol 9:191-204.

Ryall RW (1970) Renshaw cell mediated inhibition of Renshaw cells: patterns of excitation and inhibition from impulses in motor axon collaterals. J Neurophysiol 33:257-270.

Schmidt BJ, Jordan LM (2000) The role of serotonin in reflex modulation and locomotor rhythm production in the mammalian spinal cord. Brain Res Bull 53:689-710.

Severin FV, Orlovskii GN, Shik ML (1968) Reciprocal influences on work of single motoneurons during controlled locomotion. Bull Exp Biol Med 66:713-716.

Stein PS, Daniels-McQueen (2002) Modular organization of turtle spinal interneurons during normal and deletion fictive rostral scratching. J Neurosci 22:6800-6809.

Tamamaki N, Yanagawa Y, Tomioka R, Miyazaki J, Obata K, Kaneko T (2003) Green fluorescent protein expression and colocalization with calretinin, parvalbumin, and somatostatin in the GAD67-GFP knock-in mouse. J Comp Neurol 467:60-79.

Whelan P, Bonnot A, O'Donovan MJ (2000) Properties of rhythmic activity generated by the isolated spinal cord of the neonatal mouse. J Neurophysiol 84:2821-2833.

Wilson JM, Hartley R, Maxwell DJ, Todd AJ, Lieberam I, Kaltschmidt JA, Yoshida Y, Jessell TM, Brownstone RM (2005) Conditional rhythmicity of ventral spinal interneurons defined by expression of the HB9 homeodomain protein. J Neurosci 25:5710-5719.

Windhorst U (1996) On the role of recurrent inhibitory feedback in motor control. Prog Neurobiol 49:517-587.

Zar JH (1974) Biostatistical analysis. Englewood Cliffs, NJ: Prentice-Hall. 